\title{
Inter-Commodity Spread Trading Using Neural Network and Genetic Programming Techniques
}

\author{
Meng-feng Yen ${ }^{1 *}$ Tsung-nan $\mathrm{Chou}^{2}$ Ying-yue $\mathrm{Ho}^{3}$ \\ ${ }^{1}$ Graduate Institute of Finance and Banking, National Cheng Kung University, TAIWAN \\ ${ }^{2}$ Department of Finance, Chaoyang University of Technology, TAIWAN \\ ${ }^{3}$ Graduate Institute of Finance, Chaoyang University of Technology, TAIWAN
}

\begin{abstract}
We employ the methods of neural network (hereafter $\mathrm{NN}$ ) and genetic programming (hereafter GP) in this paper to construct a spread trading system, respectively, to forecast the trend of the price spread between Taiwan Stock Exchange Electronic Index Futures (hereafter TE) and Taiwan Stock Exchange Finance Sector Index Futures (hereafter TF). To forecast the trend of the spread, we use a variety of technical indicators as the inputs to our two models. We tend to long one contract and short another if the next-day return of the former is predicted to be larger than the latter. If the spread trend is predicted to change its direction, we close off the position and open a new position completely contrary to the closed one. We compare the trading performances of this momentum strategy to the day trade strategy, i.e. closing off our positions before the market close ever day. We find that the momentum strategy tends to outperform the day trade strategy and that the BackPropagation NN (hereafter BPNN) model is superior to the GP model under both strategies whilst both are profitable.
\end{abstract}

Keywords: BPNN, Genetic Programming, InterCommodity Spread, Momentum Strategy.

\section{Introduction}

Inter-commodity spread trading involves longing one futures and shorting another at the same time. The prices of their underlying products must be correlated with each other to some extent. What matters is not their individual price, but the variation of their price spread. If the variation of their price spread is predictable, we might be able to make risk-free profits. For example, if the spread is predicted to grow in one direction, we could simply long the relatively strong futures contract and short the weak one. To the contrary, if the spread is predicted to shrink or change its direction, we could close off the previous position and go into an opposite one, i.e. longing the previously weak futures contract and short the previously strong one. The literature of inter-commodity spread trading mainly involves using the cost-of-carry theory to calculate the theoretical prices of any two correlated futures contracts and examine whether their market prices are away from these calculated values. Given that the spread between their market prices will converge at the end to its theoretical value, we could form an arbitrage position by buying the relatively strong futures (the price of which is under-estimated) and shorting the relatively weak futures (the price of which is over-estimated). As long as the transaction cost could be covered by the profits from arbitraging out of this situation, we could make risk-free money. For example, Billingsley and Chance (1988) study the spread between S\&P 500 and NYSE futures for the period from 1982 through 1986. They find that the spread during their sample period is, on average, larger than its theoretical value. Due to the transaction cost, however, they do not find it profitable by trading the spread. Unlike the previous study such as above, this paper does not calculate the theoretical prices of the futures contracts using the cost-of-carry theory, but rather, forecast the direction of the spread. Namely, we forecast the difference between the returns of the two futures for one time step ahead, and trade the spread out of such forecasts.

Amongst the variety of forecasting approaches, artificial intelligence has been one of the most popular techniques in a range of areas including finance. Two of the recent successes are the neural network and genetic programming algorithms. Kimoto and Asakawa (1990), for instance, use a neural network model to find the best timing of buying stocks for the next month. They train their NN model using the fundamental economic data underlying TOPIX as the explanatory variables from Jan 1987 through Sep 1989.

\footnotetext{
*Author for correspondence: yenmf@mail.ncku.edu.tw; Tel:+886-6-2757575 ext 53443; Fax:+886-6-2744104
} 
Kim and Han (2000) add genetic algorithms approach to feature discretization and the determination of connection weights for artificial neural networks (ANNs). They find that such a practice helps speed the learning process of the network, simplify the feature space, and exclude irrelevant factors. Their empirical results tend to show that the a neural network model coupled with the GA approach to the feature discretization outperforms both the back-propagation neural network model without the use of GA algorithms and the GA-NN model with the GA applied to only the learning algorithm. The first method increases the forecast accuracy by $10 \%$ to 11 $\%$ relative to the latter two competing approaches. Jang et al. (1991) employ a dual-module neural network to develop an intelligent stock trading system for Taiwan's stock market. They use this system to forecast the short-term prices of stocks with the first module used to select which stocks to invest and the second module used to forecast the future trend of the stock prices. They then use long-term and short-term technical indicators to train their networks. They find that their neural network outperforms the benchmark model of a multi-variable linear regression.

Allen and Karjalainen (1999) search the optimal trading rule for stocks by the genetic programming method and suggest its application to other economic and micro-economic areas. Iba and Sasaki (1999) apply the genetic programming method to forecast the trend of Nikkei 225 index. They use the historical index prices to train the model and predict the future ten daily close prices of the index. According to the direction of the forecasted index movement for the next ten trading days, they simulate buying and selling the spot index and find that the forecast accuracy is as high as $60 \%$.

By forecasting the next-day relative strength between TE and TF contracts, we are able to form a corresponding position by longing one contract and shorting the other or vice versa. If the spread tends to grow in the same direction for a period of time, we can thus make profits by entering into the positions illustrated above. This paper aims to study whether it is possible to provide the traders in Taiwan's futures market with a reliable program trading system of this like by the BPNN or GP model. The rest of this paper is organized as follows. Section 2 documents the data and explanatory variables. Section 3 discusses the models and investment strategies. Section 4 examines the results and Section 5 concludes.

\section{Data and Predictor Variables}

We collect daily data for the two index futures contracts from the Taiwan Economic Journal database
(TEJ database) for the period of 18 December 2003 through 22 December 2005. In total, we have 500 daily data across 26 months for each contract. To train our two models, we divide each series of data into a training period from 18 December 2003 to 16 March 2005 (306 daily data) and a forecast evaluation period from 17 March 2005 to 22 December 2005 (194 daily data).

Inspired by the literature of stock price forecasting, we use a backward stepwise regression to screen some thirty technical indicators to select the following predictor variables: 9-day raw stochastic value of TE price, 9-day Williams \%R of TE price, daily trading volume and daily open interest of both $\mathrm{TE}$ and $\mathrm{TF}$, daily return of TF, bias from the 3-day exponential moving average of TF price (3-day bias), variation percentage of the daily open interest of TF, basis of both TE and TF, and whether the difference of the 3day bias of TE from its TF counterpart is positive (dummy $=1$ if yes and 0 if otherwise).

\section{Models and Investment Strategies}

\subsection{BPNN and GP}

Given the success of the BPNN model in the literature of $\mathrm{NN}$ forecasting, we employ this widely-used model to forecast our TE-TF spread. The output variable is defined as $(1,0)$ if the next-day return ${ }^{1}$ of TE is larger than that of TF, and $(0,1)$ otherwise. To obtain the best structure of our BPNN model, we employ the genetic algorithm to optimize the number of hidden layers and the number of processing units (neurons). We find that the best number of hidden layers is 2 , with 15 nodes in the first hidden layer and 18 in the second. Other parameterization specified with Matlab 6.5 includes: transfer function $=$ sigmoid function, learning rate $=$ 0.7 , momentum coefficient $=0.1$, and number of learning iteration $=1000$. Further details are omitted here.

Turning to our GP model, it is an extension of the genetic algorithm developed by Koza (1992). The output variable is the next-day return of TE minus that of TF. For a controllable calculation, we set the population size equal to 50 . Other parameterization includes MSE as the fitness function, 100 generations at maximum, selection by roulette wheel by rank, onepoint crossover with a crossover rate of 0.2 , multiplepoint mutation with a mutation rate of 0.04 . We perform the coding with Matlab 6.5.

\footnotetext{
${ }^{1}$ The next-day return refers to the difference of close price from the open price divided by the open price of the next trading day.
} 


\subsection{Trade and Momentum Strategies}

Day Trade Strategy:

This strategy involves trading every day according to the predicted results of the two models. For instance, when the output of our BPNN model is close to $(1,0)$, we long TE and short TF upon the open of the futures market the next trading day and vice versa. We close off this position upon the market close, irrespective of making profit.

Momentum Strategy:

Unlike the day trade strategy, we do not necessarily close off our position upon the market close of the next day. We generate forecasts for the next-day spread after the market close of every trading day. If we come up with a spread forecast of the same direction as in the present day, we hold the position until the spread is predicted to change its direction. For example, if $(1,0)$ on day $t$ switches to $(0,1)$ on day $t+1$ by our BPNN model or $0.3 \%$ (return of TE minus return of TF) on day $t$ switches to $-0.2 \%$ on day $t+1$, we close the original position and construct a new position by shorting a TE contract and longing a TF contract upon the market open of the next day. This is what we call as the momentum strategy.

Taking into account transactions costs consisting of commissions for the brokers and transaction taxes, we deduct 1,200 NT dollars from the profit or loss resulting from the offset of every position. Please refer to http://www.taifex.com.tw for the details of the latest transaction tariff, margin requirements, delivery date, etc.

\section{Results and Analyses}

We evaluate the forecast performance of these two models by both statistic and economic loss functions, i.e. the percentage of correct predicted sign of the spread and profitability, respectively.

\subsection{Predicting Accuracy}

We use the percentage of correct sign forecasts of the TE-TF spread to evaluate the forecast accuracy of both models. The results of Tables 1.1 and 1.2 below tend to suggest that the percentage of correct predicted signs of the TE-TF spread for both models is over $50 \%$, the BPNN model being better than the GP model In particular, the average rate of accuracy for the the BPNN model is nearly $60 \%$, meaning 6 profitable transactions out of every ten. Such a level of forecasting accuracy would give us some confidence in trading the spread in the long run as long as we stick to the model forecasts in trading.

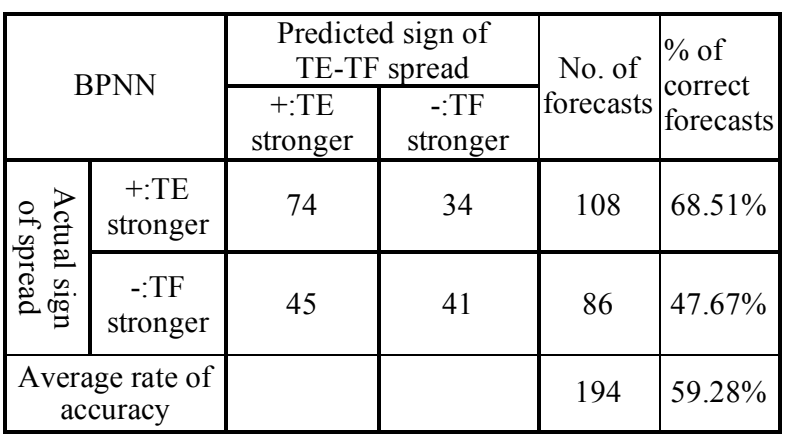

Table 1.1: Actual vs. BPNN-Predicted Signs of the TE-TF spread

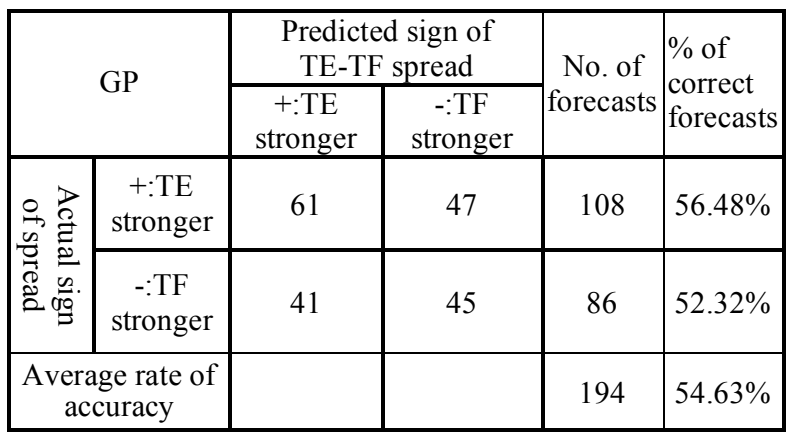

Table 1.2: Actual vs. GP-Predicted Signs of the TE-TF spread

\subsection{Profitability}

The result of Table 2 below suggests that both models are profitable during our forecast horizon from 17 March 2005 to 22 December $2005^{2}$. However, the BPNN model tends to perform not only more profitably but also more steadily than the GP model. Trading according to the BPNN model leads to a loss in only one month under both strategies. The loss incurred by the BPNN model in either August 2005 (under the day trade strategy) or April 2005 (under the momentum strategy) is almost nothing relative to the profits it makes over the other months. On the contrary, the GP model brings losses in four months under both strategies. The size of these losses under both strategies is not negligible relative to its counterpart of those profits. This result tends to suggest the reliability of the BPNN model relative to the GP model. In addition, the momentum strategy is clearly superior to the day trade strategy. This is in line with intuition since we undertake fewer transactions and incur thus less transaction cost, but allow profitable positions to pile up profits.

\footnotetext{
${ }^{2}$ The original margin requirement is NT\$ 90,000 for a TE contract and NT\$ 75,000 for a TF contract, amounting to NT\$ 165,000 in total. Taking into account the optimization of the original margins, however, would reduce the margin requirement to only NT\$90,000, which nearly doubles those rates of return in Table 2 .
} 


\begin{tabular}{|c|c|c|c|c|}
\hline \multirow{3}{*}{ Delivery Month } & \multicolumn{4}{|c|}{ Model } \\
\hline & \multicolumn{2}{|c|}{$\begin{array}{c}\text { Profits (NT\$) by } \\
\text { BPNN }\end{array}$} & \multicolumn{2}{|c|}{ Profits (NT\$) by GP } \\
\hline & Day Trade & Momentum & $\begin{array}{l}\text { Day } \\
\text { Trade }\end{array}$ & Momentum \\
\hline Apr & 400 & -12200 & 146800 & 159400 \\
\hline May & 84800 & 29000 & -54000 & -10200 \\
\hline Jun & 29800 & 31200 & 45800 & 44800 \\
\hline Jul & 60600 & 94800 & -19000 & -45600 \\
\hline Aug & -1400 & 18000 & 12600 & 57200 \\
\hline Sep & 74800 & 83000 & 14800 & 8200 \\
\hline Oct & 15800 & 25800 & -24600 & -10200 \\
\hline Nov & 10800 & 28200 & 45600 & 74600 \\
\hline Dec & 13200 & 32000 & -52800 & -32400 \\
\hline \begin{tabular}{|c|} 
Total Profits \\
(in NT dollars)
\end{tabular} & 288800 & 329800 & 115200 & 245800 \\
\hline $\begin{array}{c}\text { Number of } \\
\text { Transactions }\end{array}$ & 194 & 52 & 194 & 60 \\
\hline $\begin{array}{c}\text { Cumulative rate } \\
\text { of return }\end{array}$ & $175.03 \%$ & $199.88 \%$ & $69.82 \%$ & $148.97 \%$ \\
\hline $\begin{array}{c}\text { Annualized rate } \\
\text { of return }\end{array}$ & $233.37 \%$ & $266.51 \%$ & $93.09 \%$ & $198.63 \%$ \\
\hline $\begin{array}{c}\text { Average rate of } \\
\text { return per } \\
\text { transaction }\end{array}$ & $0.90 \%$ & $3.84 \%$ & $0.36 \%$ & $2.48 \%$ \\
\hline
\end{tabular}

Table 2: Profitability of both models under both strategies across the forecasts horizon from 17 March 2005 to 22 December 2005.

\section{Concluding Remarks}

This work aims to answer this question: is it possible to find a reliable system to trade the spread between the prices of the two of the three most popular index futures, i.e. the Taiwan Stock Exchange Electronic Sector index futures and the Taiwan Stock Exchange Finance Sector index futures? We find that using both the back-propagation neural network and the genetic programming models leads us to a profit during the sample period. However, the BPNN model is superior to the GP model in terms of both the rate of forecast accuracy and the number of profitable months. The BPNN model correctly predicts the sign of the spread to an average of nearly $60 \%$ of all of transactions, which is about $6 \%$ higher than the GP model. The BPNN model brings about profits across all but one month under both strategies, the loss incurred to the only month being very small. The GP model ends up with four months of loss under both strategies, however, which is indicative of its inability to consistently track the spread correctly. Moreover, the momentum strategy performs favorably against the day trade strategy, the former leading to a larger profit than the latter. This result suggests that the spread between TE and TF tends to demonstrate a characteristic of 'momentum.' Stated otherwise, the strength of the TE-TF spread of either sign tends to grow for a period time before the spread changes its direction. This fact also suggests an arbitrage opportunity. The main thrust of this research tends to suggest that the BPNN model under the momentum strategy is the best choice amongst all four methods investigated from the perspective of inter-commodity spread trading.

Further research could follow this line to investigate whether there are indeed arbitrage opportunities from trading inter-commodity spreads in Taiwan's futures market by extending the sample period and including more underlying indexes. Also, most of the technical indicators used as our explanatory variables originate from either the close price or the trading volume. Amongst others, however, the open interest of large futures traders, the movement of market money of all participants from one industry to another, the price of the leading stock in each industry of the Electronic and Finance sectors, etc. might also exert impact on the spread. It is therefore warranted to include more explanatory variables of this sort when applying the stepwise regression.

\section{References}

[1]F. Allen and R. Karjalainen, "Using Genetic Algorithms to Find technical Trading Rules," Journal of Financial Economics, pp. 245-271, 1999.

[2] R. S. Billingsley and D. M. Chance, "The Pricing and Performance of Stock Index Futures Spreads," The Journal of Futures Markets, pp. 303-328, 1988.

[3] H. Iba and T. Sasaki, Using Genetic Programming to Predict Financial Data, Proceedings of the Congress on Evolutionary Computation, pp. 244251, 1999.

[4] G. Jang, F. Lai, B. Jiang, and L. Chien, "Intelligent Stock Trading Decision Support System with Price Trend Prediction and Reversal Recognition Using Dual-Module Neural Networks," Journal of Applied Intelligence, pp. 225-248, 1993.

[5] K. Kim and I. Han, "Genetic Algorithms Approach to Feature Discretization in Artificial Neural Networks for The Prediction of Stock Price Index", Expert Systems with Applications, pp.125-132, 2000.

[6] T. Kimoto and K. Asakawa, Stock Market Prediction System with Modular Neural Networks, International Joint Conference on Neural Networks, pp. 1-6, 1990. 